\title{
Transitional errors and deep structure differences'
}

ROBERT E. DAVIDSON, Research and Development Center for Cognitive Learning. The University of Wisconsin, Madison, Wis. 53706

Sentences which have the same description in surface structure may have very different representations in deep structure where the actual grammatical relationships are specified. For two experiments, independent groups of Ss learned lists of sentences which were identical in surface structure but different in deep structure. Error scores for different word-to-word transitions indicated that transitional error probabilities detected different recall patterns reflecting the deep structure differences. Operational tests of this sort should aid the psycholinguist in constructing psychological competence models for language behavior.

Recent linguistic theory postulates a "deep structure" to any sentence in the language (Chomsky, 1965). The deep structure description marks the actual grammatical relationships that exist in the sentence. Two sentences which are marked differently in their underlying structure might give rise to sentences which have the same constituent descriptions in "surface structure." Thus, while two sentences may be the same in surface structure, i.e.,

(1) Delicate lace was produced by tailors,

(2) Delicate lace was produced by hand,

the grammatical relations that exist in deep structure are markedly different. In Sentence 1 tailors actually serves as the logical subject, and the passive sentence can be made active: Tailors produced delicate lace. In Sentence 2 hand is actually a part of a manner adverbial modifier, and the subject of the sentence is not stated.

Psycholinguistic research has attempted to demonstrate that if Ss are responsive to deep structure when they process sentences, then they should show behavioral differences in some kind of appropriate task. For example, Fodor \& Garrett (1967) found that relative pronoun cues in surface structure made sentences easier to understand because such cues signaled important relationships in deep structure. Also, the research of Blumenthal (1967) and Blumenthal \& Boakes (1967) indicates that, under certain conditions, the number of sentences recalled is more a function of deep structure than it is of surface structure descriptions. The work of Savin \& Perchonock (1965) suggests that sentences may be coded in memory in a way that parallels their deep structure descriptions.

On the other hand, the surface description of sentences seems to be the more important determiner in perceptual tasks such as speech perception (Fodor \& Bever, 1965) or reading (Mehler, Bever, \& Carey, 1967). However, the basically nonperceptual experiments of sentence learning over trials, in which the number of transitional errors between adjacent words in the sentence was the dependent measure, have consistently shown that error patterns follow the constituent structure at the surface (Johnson, 1965). Certainly a sentence-learning task requires the processing of sentences in memory, and the question that arises is whether transitional error probability (TEP) patterns will be responsive to the deep structure differences in sentences that have the same surface structure.

\section{EXPERIMENT 1}

Subjects

Forty students at the University of Wisconsin served as Ss. Materials

Ten sentences of the manner adverbial type (MA) and 10 of the full passive type (FP) were constructed. Examples:

MA Important messages were dispatched by wire. FP Important messages were dispatched by governments.

Notice that the constituent phrase structure at the surface is the same for both types of sentence. The structure was always of the form: adjective-noun-was/were-verb-by-noun. The sentences were recorded on tape. The reading time for each was approximately $5 \mathrm{sec}$, with a slight pause between sentences.

\section{Procedure}

Small groups of Ss (four to eight in each group) listened to either MA or FP sentences over five learning-test trials. For each trial, the $S$ heard a different random arrangement of the 10 sentences. After the last sentence was read for the learning portion of the trial, the Ss were tested for their recall. They were given $3 \mathrm{~min}$ to write down the words they could remember. Booklets with five pages (trials) were provided, and each page contained 10 separate lines with six dashes to the line. The Ss were encouraged to guess words and their locations in sentences.

\section{Scoring}

Three different types of scores were computed for each $S$. The first of these was the number of words correct at the constituent word level, i.e., adjective ... noun ... main verb ... noun [only the main verb was scored because of the constant ( . . . was/were . . . by . . . ) pattern]. The constituent word scores were summed over the 10 sentences for each trial. Thus, four scores for the number of adjectives, main verbs, etc., were computed for each $S$ at each trial.

A second type of score, the TEP, was computed for each $S$. The TEP for each left-to-right, word-to-word [again ignoring the ( . . was/were ... by)] transition within each sentence was determined by dividing the frequency that a word following a transition was incorrect by the frequency that the word before the transition was correct. That is, for the first transition (adjective ... noun) in the example sentences, the TEP would be the frequency that messages was incorrect, given that important was correct, divided by the frequency that important was correct. There are three transitions in the test sentences. They have the following pattern: adjective to noun (TEP1), noun to main verb (TEP2), main verb to noun (TEP3). This last noun is, in fact, the logical subject in FP but is part of the adverbial modifier in MA. A TEP for each transition was obtained by summing over the sentences and over trials for each $S$.

A third type of score computed for each $S$ was the DTEP. It was computed by the TEP procedure, but here the transitions were ordered differently. A DTEPI score was for the transition between the logical-subject noun (or the manneradverbial noun in MA sentences) and the main verb. A DTEP2 transition existed between the main verb and adjective, and a DTEP3 transition was between adjective and noun [e.g., Governments (DTEP1) dispatched (DTEP2) important (DTEP3) messages ]. Note that the TEP1 and DTEP3 represent the same adjective-to-noun transition.

The justification for the DTEP score is that it should be sensitive to the passivization transformation that intimately links FP passive sentences and their active representation to some deeper structure. Similar links do not exist between MA passives and some active representation, or between these MA sentences and some deeper structure which the DTEP score would reflect.

\section{RESULTS}

Analysis of variance of the words-correct measure showed 
Table 1

Transitional Error Probabilities for Each Type of Sentence and Type of Transition

\begin{tabular}{lcccc}
\hline & & \multicolumn{3}{c}{ Transition Number } \\
\cline { 3 - 5 } Transition Type & Sentence Type & 1 & 2 & 3 \\
\hline \multirow{2}{*}{ TEP } & MA $^{\mathrm{a}}$ & .0992 & .2514 & .1029 \\
& FPb $^{*}$ & .0939 & .2309 & .1021 \\
\multirow{2}{*}{ DTEP } & MA $^{\mathbf{c}}$ & .2749 & .1211 & .0992 \\
& FP $^{\mathbf{b}}$ & .1944 & .1361 & .0939 \\
\hline
\end{tabular}

a Important messages dispatched wire.

$b$ Important messages dispatched governments.

c Wire dispatched important messages.

d Governments dispatched important messages.

differences in recall for the various constituent words $(F=24.24, \mathrm{df}=3 / 114, \mathrm{p}<.001)$ and, of course, for trials. The mean number of words correct per trial at each constituent word level was: adjective $=6.29$, noun $=6.45$, main-verb $=5.50$, noun $=6.42$. A multivariate analysis of variance for the more interesting contrast between the sentence-type groups (MA vs FP) indicated no difference $(\mathrm{F}=1.15, \mathrm{df}=4 / 187)$.

Table 1 presents the mean transitional error probabilities for this experiment.

Tests among the error scores at each transition point were not carried out. Typically, significant differences are found (Johnson, 1965). Of interest to this study are tests between the sentence-type groups for each transition point. Of particular interest are the TEP3 and the DTEP1 transitions. Mann-Whitney tests indicated no differences at any transition for the TEP scores. This result was not entirely unexpected since the surface structure of the two types of sentences do not differ. However, the most critical left-to-right transition in surface structure is that of TEP3 which moves from main verb to manner adverbial noun or to logical subject in the MA and FP sentences, respectively. A test of the TEP3 transition produced a nonsignificant $U$ of 192.5 .

For the DTEP scores, however, the MA and FP groups differ for DTEP1. A normal approximation to the $U$ statistic was significant $(Z=2.69, p<.004)$. It appears that $S s$ recall the same number of words, but the pattern of recall is different for the two groups.

\section{EXPERIMENT 2}

Subjects

Sixty students at the University of Wisconsin served as Ss. Materials

The tapes of Exp sriment 1 were used. Procedure

Essentially the same procedure of Experiment 1 was used here. The booklets were changed to meet design requirements. Design

Four independent groups were established by providing two different sentence types (MA, FP) and cues (first noun, second noun). Depending upon his group assignment, the $S$ received a booklet which provided cue words for each sentence at every trial. Either the first noun of the sentence or the second noun of the sentence was printed. The Ss were encouraged to use the cue words to aid them in recall. This design is, in gross outline, that of Blumenthal (1967). Predictions were similar. That is, the Ss serving under the FP condition and who received the second noun (logical subject) as a cue were expected to be facilitated in their recall.
Scoring

With some necessary changes, the three types of scores computed in Experiment 1 were calculated here. Because different cue words were provided, word constituent scores were not meaningful. Instead, the number-of-words-correct score was a summation of the three remaining words in each of the 10 sentences for each trial; these were cast in a 2 by 2 by 5 analysis of variance. The TEP score and DTEP score were determined as before. It can be noted that either larger or nonexistent error probabilities for some cells would occur because cue words might constitute one side or the other of a transition.

\section{RESULTS}

Analysis of the words-correct measure revealed a significant Cue effect $(F=4.59$, df $=1 / 56, p<.05)$ with the first noun providing greater facilitation of recall. Neither the Sentencetype effect $(F=2.28$, df $=1 / 56)$ nor the predicted interaction (F $<1.00$ ) was found to be significant.

In some conditions, the cues that were provided made up the right-hand side of the transition; therefore, certain transitional errors did not exist. But despite this general limitation, the critical transitions could be tested. Again, the tests of interest contrast the MA and FP groups. On Mann-Whitney tests, only the DTEP1 transition scores were significantly different $(Z=2.57, p<.005)$ with $M A=.3474$ and $\mathrm{FP}=.2869$.

\section{DISCUSSION}

Transitional error patterns have consistently shown themselves to be sensitive to sentence surface structure differences. The results of these experiments suggest that the metric may be very sensitive to deep structure differences as well. Of course, the addition or elaboration of any good operational test to the study of language is especially needed at this time. The psycholinguistic researcher has reached the point where he no longer is content merely to demonstrate the verities of a linguistic competence model; instead, he is ready to look at language behavior differently than he has in the past. He is ready to postulate psychological competencies with respect to language that are likely to be, at the same time, at variance with any fully elaborated system of linguistic operations and with any traditional psychological theory as well.

\section{REFERENCES}

BLUMENTHAL, A. Promoted recall of sentences. Journal of Verbal Learning \& Verbal Behavior, 1967, 6, 203-206.

BLUMENTHAL, A., \& BOAKES, R. Prompted recall of sentences, a further study. Journal of Verbal Learning \& Verbal Behavior, 1967, 6, 674-767.

CHOMSKY, N. Aspects of the theory of syntax. Cambridge: M.I.T. Press, 1965.

FODOR, J., \& BEVER, T. The psychological reality of linguistic segments. Journal of Verbal Learning \& Verbal Behavior, 1965, 4, 414-420.

FODOR, J., \& GARRETT, M. Some syntactic determinants of sentential complexity. Perception \& Psychophysics, 1967, 2, 289-296.

JOHNSON, N. F. The psychological reality of phrase-structure rules. Journal of Verbal Learning \& Verbal Behavior, 1965, 4, 469-475.

MEHLER, J., BEVER, T., \& CAREY, P. What we look at when we read. Perception \& Psychophysics, 1967, 2, 213-218.

SAVIN, H., \& PERCHONOCK, E. Grammatical structure and the immediate recall of English sentences. Journal of Verbal Learning \& Verbal Behavior, 1965, 4, 348-353.

\section{NOTE}

1. A part of this research was supported by Contract OE 5-10-154 with the United States Office of Education. Portions of the paper were presented at the annual meeting of the American Psychological Association, 1968, San Francisco. 